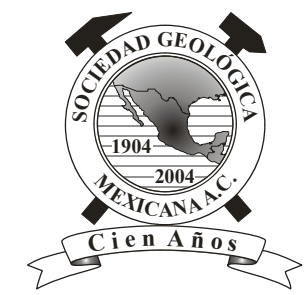

\title{
Estimación de la respuesta térmica de la cuenca lacustre del Valle de México en el siglo XVI: un experimento numérico
}

\author{
Angel Ruiz-Angulo ${ }^{1, *}$, Erika Danaé López-Espinoza ${ }^{1}$ \\ ${ }^{1}$ Centro de Ciencias de la Atmósfera, Universidad Nacional Autónoma de México. Ciudad Universitaria, 04510 México, D.F., \\ México. \\ *angel@atmosfera.unam.mx
}

\section{Resumen}

Se presenta un estudio de la respuesta térmica para los meses de enero - febrero y agosto - septiembre en un escenario que incluye un cuerpo de agua representando el sistema lacustre del Valle de México, y excluye la cobertura urbana. El experimento numérico tiene como objetivo replicar el conjunto de lagos que conformaban el sistema lacustre hace 500 años. Las simulaciones numéricas se realizaron con el modelo Weather Research and Forecasting (WRF) considerando un escenario con las condiciones de uso de suelo actuales y otro con el sistema lacustre sintético, ambos escenarios empleando condiciones iniciales y de frontera de 2012. Se comparó la temperatura en superficie de ambas simulaciones y ocho estaciones meteorológicas localizadas en el Valle de México. Los resultados muestran un impacto térmico significativo en el tiempo meteorológico generado por la presencia del cuerpo de agua. Regionalmente, se observó un incremento de temperatura en superficie asociado a dos procesos simultáneos: la extinción del sistema lacustre y el crecimiento urbano. Además de la amplitud, se observa un corrimiento en el tiempo del máximo en temperatura asociado con los cambios en flujos de calor. El incremento promedio de la temperatura llega a ser mayor que el asociado al cambio climático global durante el mismo periodo de tiempo.

Palabras clave: micro clima urbano, modelación numérica, tiempo meteorológico, cuenca lacustre del Valle de México, temperatura en superficie.

\begin{abstract}
This manuscript presents a numerical study of the thermal response for the months of January-February and August-September in a scenario that includes a water body representing the lake system of the Basin of Mexico excluding the urban coverage. The numerical experiment aims to replicate the set of lakes that formed the lacustrine system 500 years ago. Numerical simulations were performed with the Weather Research and Forecasting model (WRF) considering a scenario with the current land usage conditions and one with the synthetic lacustrine system, both scenarios using initial and boundary conditions from 2012. The surface temperature from both simulations and eight meteorological stations located in the Valley of Mexico were compared. The results show a significant thermal impact on the weather generated by the presence of the lake. Regionally, an increment on the surface temperature was observed associated with two simultaneous processes: the extinction of the lacustrine system and the urban growth. In addition to the amplitude, a shift on the maximum temperature time is observed associated with changes in heat fluxes. The average increase in temperature becomes higher than that associated with global climate change over the same period of time.
\end{abstract}

Keywords: urban microclimate, numerical simulation, weather, basin of Mexico, surface temperature. 


\section{Introducción}

En la época prehispánica la existencia de un vasto lago en la cuenca endorreica del Valle de México propició una cadena de asentamientos humanos con desarrollos urbanos desde hace ya más de 2000 años (Tolstoy et al., 1977; Ezcurra, 1990a; Ezcurra, 1990b). Los asentamientos fueron ocupando la mayor parte del área de lago, y a su vez lo fueron disminuyendo drásticamente (Armillas, 1971; Musset, 1991). Durante los últimos 100 años, la población en la cuenca que abarca el Estado de México y el Distrito Federal ha crecido 17 y 14 veces más, respectivamente (INEGI, 2000). Así, el crecimiento de la población en la cuenca del Valle de México se asocia directamente con la desecación de la misma (Barragán, 1998). Durante los últimos 700 años, los cambios de cobertura y uso de suelo en la cuenca lacustre propiciados principalmente por causas antropogénicas han tenido un impacto considerable en el micro clima del Valle de México (García, 1974). Diversos estudios realizados regionalmente en el Centro de México han comprobado tanto con observaciones como con modelación numérica un incremento en la temperatura de hasta $2{ }^{\circ} \mathrm{C}$ (Jazcilevich et al., 2000; Jáuregui, 2004). Además, pueden encontrarse diversos estudios sobre paleoclimas utilizando registros ambientales naturales para describir cómo era el clima en la región central del Valle de México (Heine, 1973; Klaus, 1973). A diferencia de los estudios mencionados anteriormente, este trabajo busca tener un mejor entendimiento de las condiciones meteorológicas de hace 500 años realizando un análisis de la respuesta de temperatura en el Valle de México emulando las condiciones de la cuenca del siglo XVI mediante el uso del modelo numérico de mesoescala Weather Research and Forecasting (WRF). El experimento consistió en dos simulaciones: un experimento de control con condiciones actuales (no lagos) y otro incluyendo un único cuerpo lacustre que representa al conjunto de lagos que formaban el sistema lacustre del Valle de México (lagos). Se analizaron estas simulaciones junto con observaciones de temperatura de ocho estaciones meteorológicas localizadas en el Valle de México.

\section{2. Área de estudio}

La cuenca del Valle de México está localizada en la Planicie Central, con una elevación promedio de 2230 a $2250 \mathrm{msnm}$, rodeada por cordilleras montañosas (Sanders et al., 1979; Lozano-García et al., 1993). El sistema de lagos, estaba conformado por los lagos de Texcoco, Xochimilco, Chalco, Xaltocan y Zumpango. Los lagos de Xochimilco y Chalco estaban formados con aguas dulces mientras que las aguas de Texcoco, Zumpango y Xaltocan eran salobres. El área total del sistema lacustre durante el siglo XVI era de $1500 \mathrm{~km}^{2}$ (Ezcurra, 1990b; Jazcilevich et al., 2000; Endfield, 2008); mientras que la profundidad en promedio era de $1.5 \mathrm{~m}$ (Niederberger, 1987) con variaciones de 1 hasta 11 metros (Raynal-Villasenor, 1987; Lozano-García et al., 1993). Las continuas modificaciones antropogénicas realizadas sobre el sistema lacustre, por los asentamientos humanos desde los aztecas, la colonia y etapas posteriores, han resultado en que actualmente se conserve únicamente el $1.33 \%$ de la superficie original (Musset, 1991). En la época actual de ese enorme lago sólo existen remanentes y depósitos artificiales como El Caracol y Nabor Carrillo (Figura 1). Actualmente predominan dos tipos de climas según la clasificación de Köppen modificada por García, (1998): semiseco templado y templado subhúmedo con lluvias en verano. El área registra una temperatura media anual entre $14^{\circ} \mathrm{C}$ y $15^{\circ} \mathrm{C}$, con precipitación media anual de 900 a $1000 \mathrm{~mm}$, correspondiendo casi la totalidad de la lluvia a los meses de junio a octubre y una humedad relativa promedio de $60 \%$ (INE, 2014). A inicios del siglo XX, los tipos de vegetación que predominaban eran los pastizales, matorrales, tierras de cultivo y tierras áridas inhabitadas (USGS, 1993).

\section{Materiales y métodos}

\subsection{Configuración del modelo WRF}

Para realizar las simulaciones de este estudio se empleó la versión 3.6 del modelo WRF. El WRF es un sistema de simulación atmosférica y predicción numérica del tiempo creado en los 1990's capaz de simular escalas meteorológicas desde decenas de metros hasta cientos de kilómetros. Para este estudio las simulaciones numéricas emplearon un anidamiento que cubre el Valle de México y sus alrededores (Figura 2) con una malla de 124 x 205 puntos con resolución horizontal de aproximadamente 7 $\mathrm{km}$ y 30 niveles verticales. El periodo de tiempo simulado consideró dos meses secos (enero y febrero de 2012) y dos meses en temporada de lluvia (agosto y septiembre de 2012). La interacción con el dominio padre, que cubre toda la República Mexicana, fue unidireccional (Kalnay, 2003). Las simulaciones se realizaron en modo pronóstico a 120 horas con salidas horarias. Para las condiciones iniciales y de frontera se utilizaron datos de las 00Z del modelo Global Forecast System (GFS) cada seis horas con una resolución espacial de un grado. Para la inicialización de la temperatura de la superficie del mar (Sea Surface Temperature - SST) se utilizaron datos diarios de alta resolución (1/12 de grado) desarrollados por el NCEP/MMAB (National Centers for Environmental Prediction / Marine Modeling and Analysis Branch)(GDAS, 2014). Se utilizó una proyección Mercator y un paso de tiempo de 120 segundos. Se parametrizaron los siguientes esquemas: el esquema Dudhia para la radiación de onda corta, el esquema Rapid Radiative Transfer Model (RRTM) para la radiación de onda larga, el esquema Kain-Fritsch para la parametrización de cúmulus y para la capa límite planetaria se usó el esquema Yonsei University (Skamarock et al., 2008). El modelo de suelo LSM (Land 


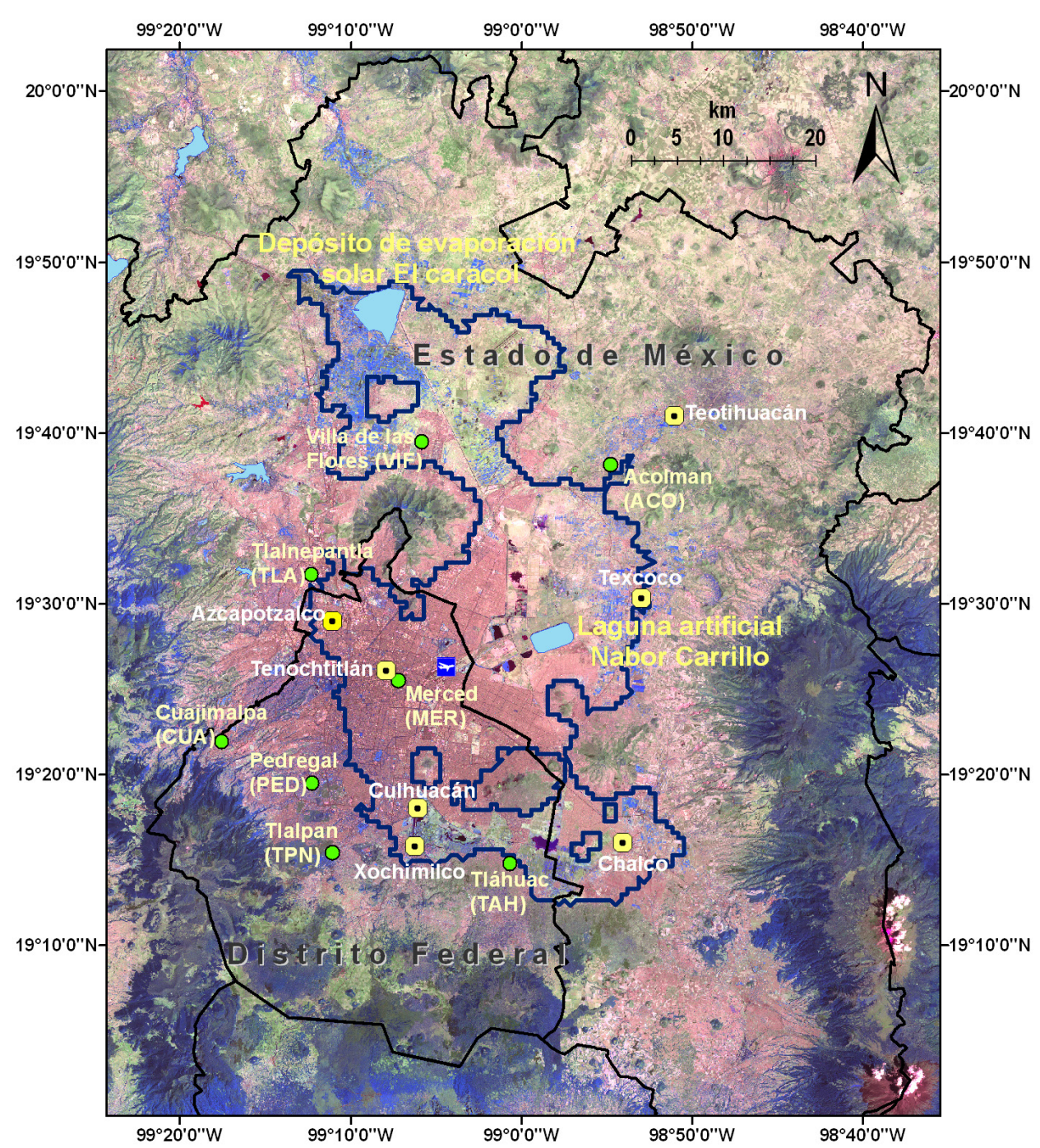

Figura 1. Imagen Landsat ETM+ del 2000 para el centro del Valle de México. El contorno azul delimita el sistema lacustre simulado, los colores en rosa sobre la imagen son la mancha urbana, los puntos verdes representan la ubicación de las estaciones meteorológicas, los puntos amarillos la localización de algunos lugares de referencia y los polígonos en azul claro los cuerpos de agua que existen actualmente.

Surface Model) empleado fue el Noah que consta de 4 capas de suelo. Los datos de uso de suelo y cobertura vegetal usados fueron los generados por el United States Geological Survey (USGS) en 1992 - 1993 con 24 clases, más una clase que define a los lagos. Se parametrizó el esquema de lagos CLM 4.5 (Community Land Model) que es un modelo de balance de energía y agua de una dimensión (Subin et al., 2012; Olsen et al., 2013) que implementa la versión actual del WRF. Este esquema ha sido validado contra observaciones y ha eliminado gran parte de los sesgos numéricos en precipitación, temperatura y flujos de calor latente (Subin et al., 2012; Thiery et al., 2013).

\subsection{Red de Meteorología y Radiación Solar}

Para estudiar la temperatura en superficie de las simulaciones, con lagos y sin lagos, se compararon contra las observaciones de ocho estaciones de la Red de Meteorología y Radiación Solar (REDMET) del Sistema de Monitoreo Atmosférico de la Zona Metropolitana del Valle de México (SIMAT) (SIMAT, 2014). El año 2012 fue seleccionado para realizar el experimento numérico ya que incluye series de tiempo de temperatura más completas para los meses de enero-febrero y agosto-septiembre. De las 16 estaciones meteorológicas que se encuentran actualmente operando se emplearon únicamente ocho que cumplían con un mínimo de $76 \%$ de datos observados (Figura 1). En la Tabla 1 se muestra información detallada para cada estación.

\section{Diseño del experimento numérico}

El experimento consistió en dos simulaciones numéricas: una de ellas considera las condiciones actuales de uso de 
suelo del modelo WRF y la otra lo modifica drásticamente. En esta última, la mancha urbana fue sustituida por un cuerpo lacustre muy similar al existente en el siglo XVI. El contorno del cuerpo fue obtenido utilizando GMT (Global Mapping Tools) con la base de datos SRTM (Shuttle Radar Topography Mission) con resolución de 90 m (Farr et al., 2007). Se seleccionó el contorno cerrado que cualitativamente fuera más representativo del sistema lacustre del siglo XVI correspondiente al nivel de $2258 \mathrm{~m}$ (Figura 3). El área total del cuerpo lacustre simulado fue de $1689 \mathrm{~km}^{2}$ con una profundidad definida constante en toda el área de 2 m (Niederberger, 1987; Raynal-Villasenor, 1987; Lozano-García et al., 1993), y la temperatura superficial del lago sintético se estimó a partir de la temperatura media en superficie diaria utilizando el nuevo esquema de lagos incluido en el WRF. En términos del índice de urbanización (ui) sugerido por Jazcilevich et al. (2000), se comparan únicamente los límites superior e inferior del índice, i.e., ui $=\{0,1\}$, equivalente a una cobertura urbana nula ( 0 con lago) o la presencia total de ella (1 sin lago). En este trabajo el índice de urbanización no consideró cambios en cobertura

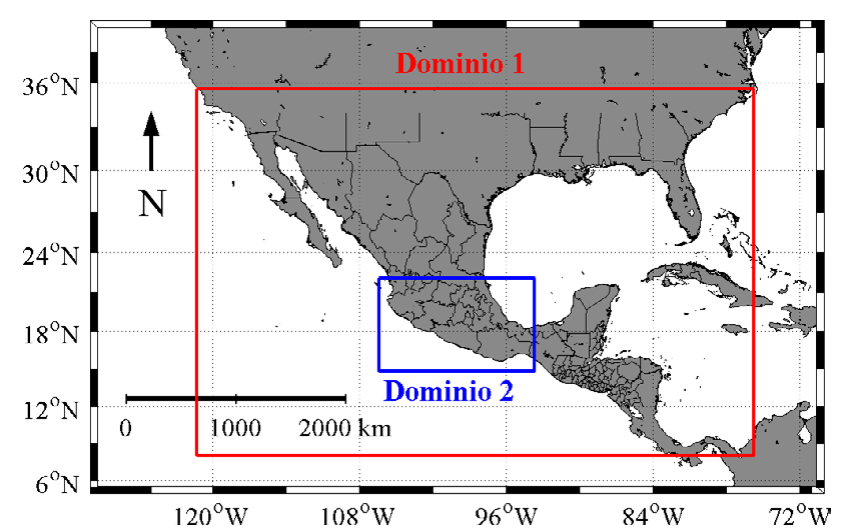

Figura 2. Dominios anidados para las simulaciones numéricas. El dominio uno abarca toda la República Mexicana con una malla de 162 x 245 puntos y una resolución horizontal de $20 \mathrm{~km}$; el dominio dos en el centro de la República con 124 x 205 puntos y con resolución horizontal de aproximadamente $7 \mathrm{~km}$. vegetal, debido a que el objetivo está enfocado únicamente a caracterizar el efecto térmico del sistema lacustre.

\section{Resultados}

Se comparó la temperatura en superficie entre las simulaciones numéricas y las observaciones de las ocho estaciones seleccionadas de la REDMET. A partir de cada una de las salidas del modelo WRF se consideraron únicamente las primeras 24 horas de simulación a partir de la hora 01:00 (tiempo local). Los resultados del pronóstico para las siguientes horas de simulación (48 a $120 \mathrm{hrs}$.) no fueron considerados debido a que no mostraban diferencias significativas en los promedios mensuales de temperatura. Para realizar las comparaciones, se seleccionó el punto de malla más cercano a la ubicación de cada una de las estaciones seleccionadas y se obtuvo el promedio considerando sus ocho vecinos, buscando de esta manera tener mejores estadísticas de comparación.

Los resultados mostrados para temperatura en superficie en los meses de enero, febrero, agosto y septiembre de 2012, incluyen la variación diurna del promedio mensual y su dispersión. En la Figura 4 se muestran las comparaciones para cada estación entre las observaciones y las simulaciones numéricas para el mes de enero; las correspondientes para febrero se muestran en la Figura 5, mientras que para agosto y septiembre se presentan en las Figuras 6 y 7 respectivamente.

En la Tabla 2 se muestra un resumen para cada estación del rango promedio y las diferencias absolutas de las temperaturas máximas y mínimas del promedio mensual para los meses secos (enero-febrero) y los meses de lluvia (agosto-septiembre). El incremento promedio en la temperatura máxima para los meses secos es de $2.78^{\circ} \mathrm{C}$, mientras que para los meses de lluvia es de $2.47^{\circ} \mathrm{C}$. Por otro lado, la diferencia media absoluta en la temperatura mínima es de $1.70^{\circ} \mathrm{C}$ para los meses secos y de $1.03{ }^{\circ} \mathrm{C}$ para los meses con lluvia. Además, se observa que las estaciones que se encuentran dentro del sistema lacustre (MER y VIF)

Tabla 1. Estaciones de la REDMET empleadas para la comparación del experimento numérico.

\begin{tabular}{|c|c|c|c|c|c|c|}
\hline \multirow{2}{*}{ Zona } & \multirow{2}{*}{ Lugar } & \multirow{2}{*}{$\begin{array}{c}\text { Delegación o } \\
\text { municipio }\end{array}$} & \multirow{2}{*}{ Estación } & \multirow{2}{*}{ Clave } & \multicolumn{2}{|c|}{ Ubicación } \\
\hline & & & & & Latitud & Longitud \\
\hline Noroeste & D.F. & Tlalnepantla & Tlalnepantla & TLA & 19.529 & -99.204 \\
\hline Noreste & D.F. & Coacalco & $\begin{array}{l}\text { Villa de las } \\
\text { Flores }\end{array}$ & VIF & 19.658 & -99.096 \\
\hline Centro & D.F. & $\begin{array}{l}\text { Venustiano } \\
\text { Carranza }\end{array}$ & Merced & MER & 19.424 & -99.119 \\
\hline Suroeste & D.F. & Cuajimalpa & Cuajimalpa & CUA & 19.365 & -99.291 \\
\hline Suroeste & D.F. & $\begin{array}{l}\text { Álvaro } \\
\text { Obregón }\end{array}$ & Pedregal & PED & 19.325 & -99.204 \\
\hline Suroeste & D.F. & Tlalpan & Tlalpan & TPN & 19.257 & -99.184 \\
\hline Sureste & D.F. & Xochimilco & Tláhuac & TAH & 19.529 & -99.204 \\
\hline Centro & Edo. Méx. & Acolman & Acolman & $\mathrm{ACO}$ & 19.635 & -98.912 \\
\hline
\end{tabular}




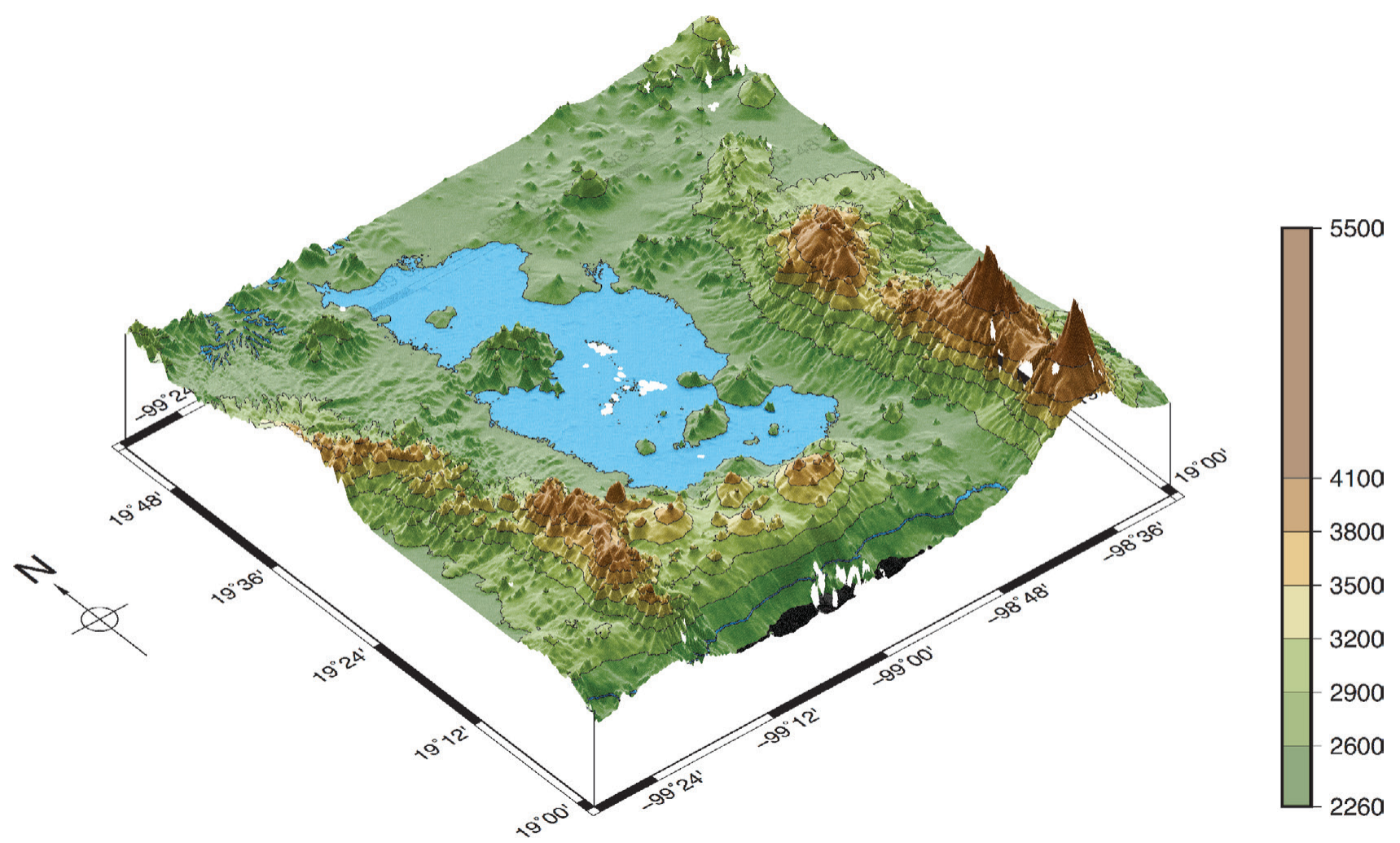

Figura 3. Topografía del Valle de México incluyendo el contorno cerrado a $2258 \mathrm{~m}$ correspondiente al cuerpo de agua utilizado en la simulación numérica con cuenca. La barra de colores muestra la elevación en metros sobre el nivel del mar (msnm).

presentan un incremento mayor en la temperatura máxima y mínima que cualquiera de las estaciones que se encuentran alrededor del lago, esto debido a la influencia directa de la presencia del cuerpo de agua. También puede observarse que, el rango promedio con la presencia del sistema lacustre es $4{ }^{\circ} \mathrm{C}$ menor que el rango promedio considerando las condiciones actuales de uso de suelo.

En la Figura 8 se presenta la distribución espacial de la anomalía de temperatura en superficie mensual considerando la diferencia entre la simulación con lagos menos la simulación sin lagos para las 12 horas del día (8:00 a 20:00 hrs) y de la noche (20:00 a 8:00 hrs). Los mapas de las anomalías se muestran para el mes de enero en época de secas y para el mes de agosto en época de lluvias. En ambos meses se observa que durante el día la temperatura sobre el lago es más fresca, mientras que por la noche la temperatura sobre el lago es más cálida.

\section{Discusión}

En relación con los cambios debidos a la variabilidad natural, e inclusive al cambio climático debido a causas antropogénicas, el impacto que conlleva la pérdida de casi la totalidad de $1500 \mathrm{~km}^{2}$ de cuerpos de agua es considerable. Aun cuando existe un gran interés en los estudios de proyecciones de cambio climático a escalas globales, los estudios del impacto antropogénico en el clima regional pasado son también de igual importancia.

El proceso del cambio de cobertura y uso de suelo en el Valle de México, principalmente por el crecimiento descontrolado de la urbanización, ha resultado en un impacto directo en las condiciones meteorológicas imperantes en el siglo XVI, cuando el sistema lacustre funcionaba como un regulador de las fluctuaciones estacionales de la temperatura. Las comparaciones puntuales entre los experimentos sin lagos y con lagos en las ocho estaciones meteorológicas estudiadas muestran un incremento promedio en la temperatura máxima para los meses secos de $2.78^{\circ} \mathrm{C}$, mientras que para los meses en época de lluvia de $2.47^{\circ} \mathrm{C}$. Con respecto a la temperatura mínima la diferencia promedio para los meses secos es de $1.70{ }^{\circ} \mathrm{C}$ y para los meses de lluvia de $1.03{ }^{\circ} \mathrm{C}$. En general, se observa en todas las estaciones un efecto de enfriamiento durante el día y calentamiento durante la noche debido a la presencia de los lagos, sin embargo dichos efectos son más evidentes en aquellas estaciones que se encuentran dentro del cuerpo de agua (MER y VIF) y aquellas que se ubican en la parte suroeste (PED y TPN) y sur (TAH) cercanas al sistema lacustre simulado. Además, a partir de los resultados observamos un desfasamiento en la ocurrencia del máximo y el mínimo de temperatura entre las dos simulaciones numéricas; sugiriendo que la rapidez de calentamiento y enfriamiento es menor en presencia del lago. 

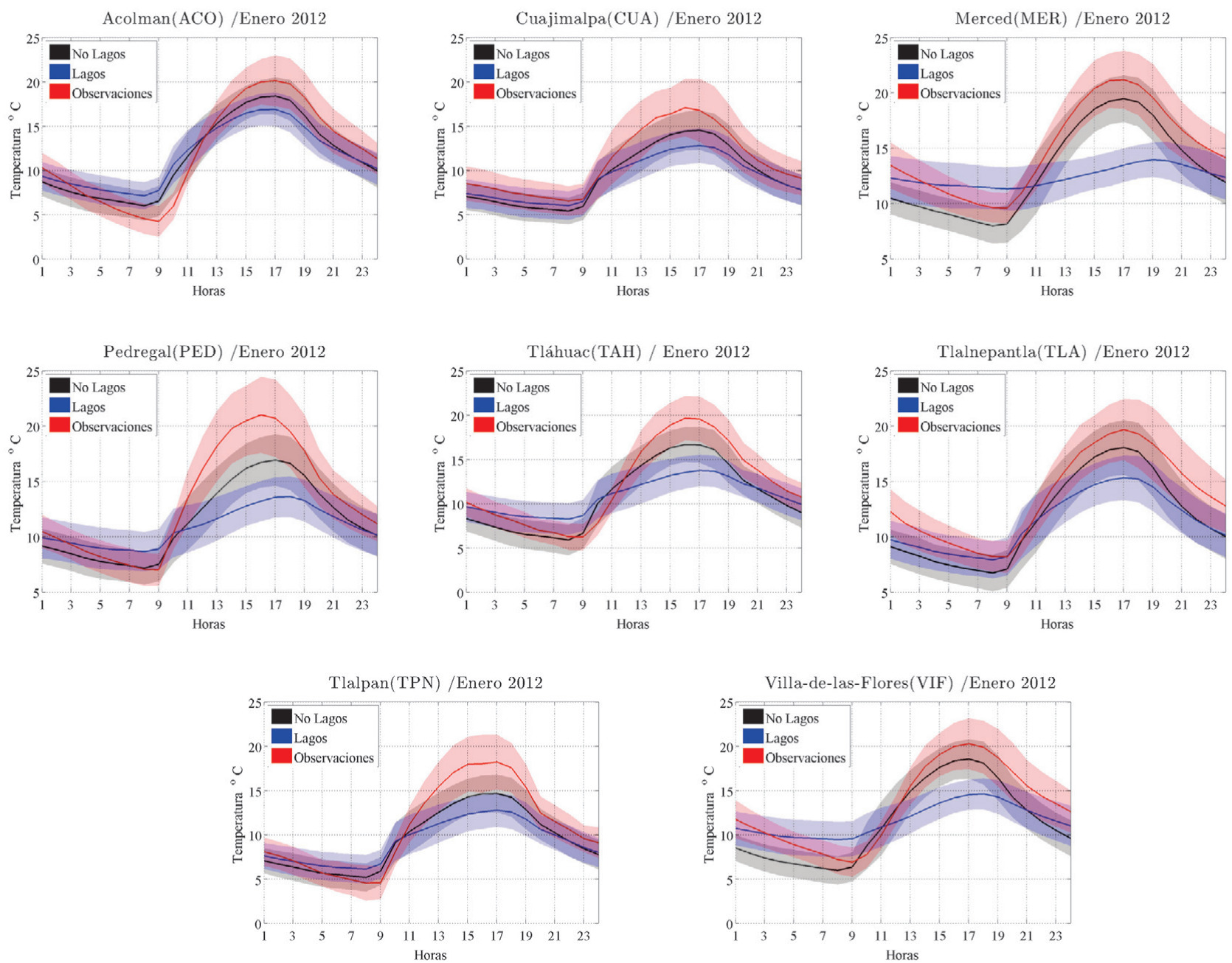

Figura 4. Comparación de las series de tiempo del promedio mensual de temperatura correspondiente al mes de enero de 2012. En color negro se muestra la simulación de control (sin lagos), en azul la simulación con lagos y en rojo las observaciones. El promedio mensual se representa con las líneas sólidas y sus dispersiones correspondientes con las bandas sombreadas.

El rango diurno de la temperatura en superficie $(\mathrm{DTR}=$ Tmáx - Tmín) para los meses secos considerando el sistema lacustre es de $5.95^{\circ} \mathrm{C}$ y de $7.41^{\circ} \mathrm{C}$ para los meses de lluvia. Sin embargo, considerando las condiciones actuales, los meses secos presentan un rango de $10.43{ }^{\circ} \mathrm{C}$ y de 10.92 ${ }^{\circ} \mathrm{C}$ en los meses de lluvia. De esta forma, observamos una mayor variabilidad de la temperatura diurna en la ausencia del sistema. Englehart y Douglas (2005) mostraron que para México el DTR está siendo significativamente positivo y por lo tanto la temperatura máxima diaria está incrementándose con mayor rapidez que la temperatura mínima diaria, como sucede en este caso de estudio. Estos cambios están asociados a los cambios regionales de cobertura y uso de suelo que han estado sucediendo en México (LópezEspinoza et al., 2012).

De lo anterior observamos que el fenómeno de degradación del clima regional en el Valle de México está ocurriendo, en parte, por el acelerado y descontrolado crecimiento de las áreas urbanas sobre la cuenca lacustre en los últimos 700 años. Regionalmente, el efecto del cuerpo de agua en latitudes medias, donde las temperaturas máximas en superficie no son despreciables (por ejemplo, en el D. F. se registran temperaturas máximas de $20.8^{\circ} \mathrm{C}$ para enero y $23.1{ }^{\circ} \mathrm{C}$ para agosto (SMN, 2014), resulta en un incremento en los flujos de calor latente en comparación con sus alrededores.

En los mapas de anomalía de temperatura, definida como Lagos menos No Lagos (Figura 8), se observa una mayor amplitud en la anomalía durante el día sobre el lago, especialmente en las regiones oeste y suroeste del sistema de lagos, asociada con un efecto de calentamiento. Estas regiones coinciden con una gran densidad en la cobertura urbana mientras que en las regiones norte y noreste, la amplitud de la anomalía es menor ya que su cobertura es principalmente vegetación y suelo descubierto. En estos mapas se observa también que durante la noche la amplitud de la anomalía es mayor en las regiones con vegetación y suelo (norte y noreste) contrario a una amplitud menor en 

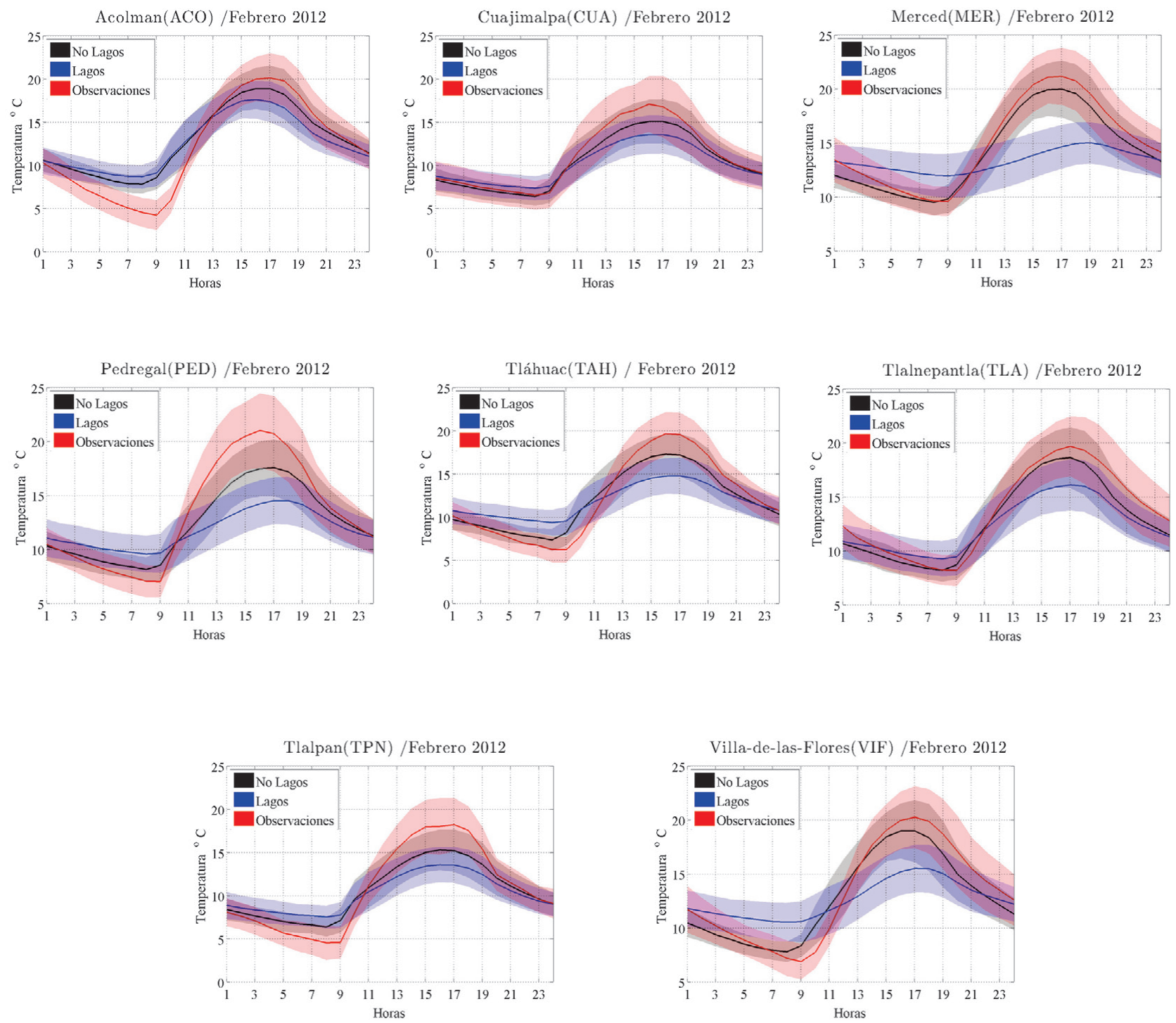

Figura 5. Comparación de las series de tiempo del promedio mensual de temperatura correspondiente al mes de febrero de 2012. En color negro se muestra la simulación de control (sin lagos), en azul la simulación con lagos y en rojo las observaciones. El promedio mensual se representa con las líneas sólidas y sus dispersiones correspondientes con las bandas sombreadas.

la región con mayor densidad de cobertura urbana (oeste y suroeste de la cuenca). Este comportamiento es debido a que la transición en el cambio de uso de suelo no ha sido homogénea para toda la región del sistema de lagos. Por un lado las regiones oeste y suroeste simulan un cambio de uso de suelo de agua a cobertura urbana, mientras que las regiones norte y noreste consideran en la simulación una transición de agua a vegetación y suelo descubierto. En la transición de agua a vegetación-suelo, el efecto de enfriamiento nocturno es más pronunciado comparado con la transición de agua a cobertura urbana, mientras el efecto de calentamiento durante el día es mayor para los casos de agua a cobertura urbana comparado con la transición de agua a vegetación-suelo (Jensen, 2007). En la simulación con lagos, el cuerpo de agua funciona como un capacitor térmico, donde existe energía térmica (calor) que es acumulada durante el día y liberada por la noche.

Obtener una línea base de la respuesta del sistema lacustre ante un escenario con condiciones actuales, ha sido posible debido a que recientemente en el 2014, se han logrado incluir dentro de los modelos numéricos de mesoescala como el WRF, esquemas de lagos realistas que ayudan a obtener resultados más confiables en temperatura, flujos de calor y precipitación (Dutra et al., 2010; Subin et al., 2012).

\section{Conclusiones}

Se presentan los resultados de la respuesta térmica de cuatro meses (enero-febrero, agosto-septiembre) de simulaciones numéricas en un escenario que incluye un 

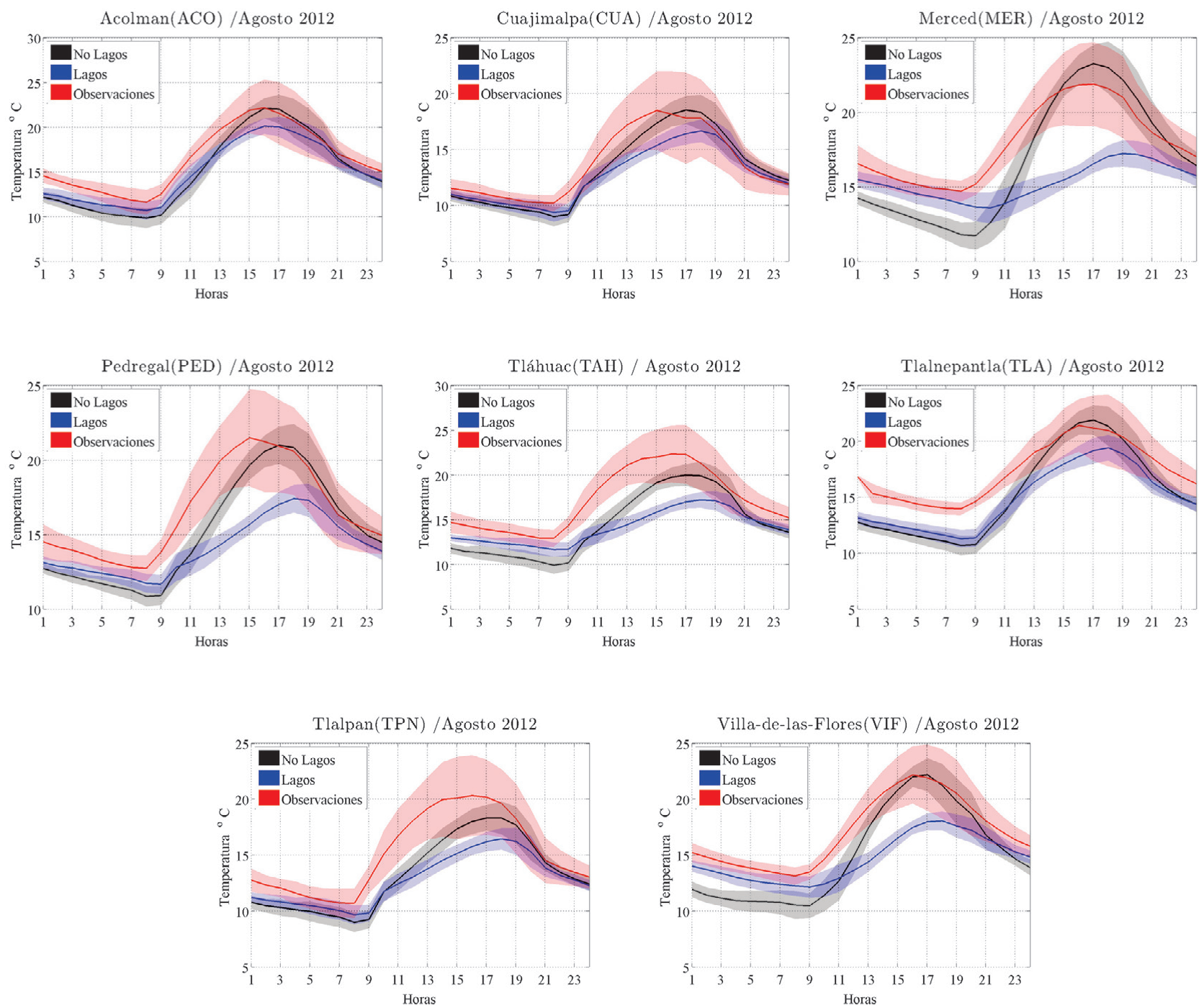

Figura 6. Comparación de las series de tiempo del promedio mensual de temperatura correspondiente al mes de agosto de 2012. En color negro se muestra la simulación de control (No Lagos), en azul la simulación con lagos y en rojo las observaciones. El promedio mensual se representa con las líneas sólidas y sus dispersiones correspondientes con las bandas sombreadas.

cuerpo de agua el cual representa el sistema lacustre del Valle de México sin urbanización y otro con las condiciones actuales de uso de suelo. El experimento numérico emula las condiciones de la cuenca hace 500 años y proporciona evidencias cuantitativas del impacto térmico ante este cuerpo de agua. La Ciudad de México se ha desarrollado de una manera no sustentable y forzosa desde sus inicios (Mancebo, 2007). En aproximadamente los últimos 500 años las modificaciones antropogénicas en el sistema lacustre del Valle de México han resultado en un incremento irreversible en la temperatura, generando un micro clima regionalmente. Los resultados de este trabajo sugieren que el impacto en la respuesta térmica debido a la extinción del sistema lacustre puede alcanzar los $5.78^{\circ} \mathrm{C}$, siendo mucho mayor que el promedio de las proyecciones globales debidas al cambio climático, i.e., $0.8^{\circ} \mathrm{C}$, en el mismo periodo de tiempo (IPCC, 2013).
Se observa que el efecto del sistema lacustre simulado regula las temperaturas regionalmente reduciendo las diferencias entre las máximas y mínimas. También se observa que el impacto que existe en el incremento de temperatura debido al crecimiento de la mancha urbana en el Valle de México ocurre a un nivel de escalas de ciudad (subregional: Distrito Federal y Ciudad de México) sugiriendo que el efecto del sistema lacustre no se extiende en una escala mayor que involucre al Centro de México y mucho menos a toda la República Mexicana. Las variaciones térmicas debidas a la presencia del sistema lacustre simulado son atribuidas principalmente a la modificación regional de la cuenca, aun cuando la cobertura de vegetación cercana no se haya modificado.

Ante el inminente cambio climático, regionalmente, las consecuencias de un cambio drástico en el uso del suelo pueden agravarse (Lankao, 2010). Este experimento 

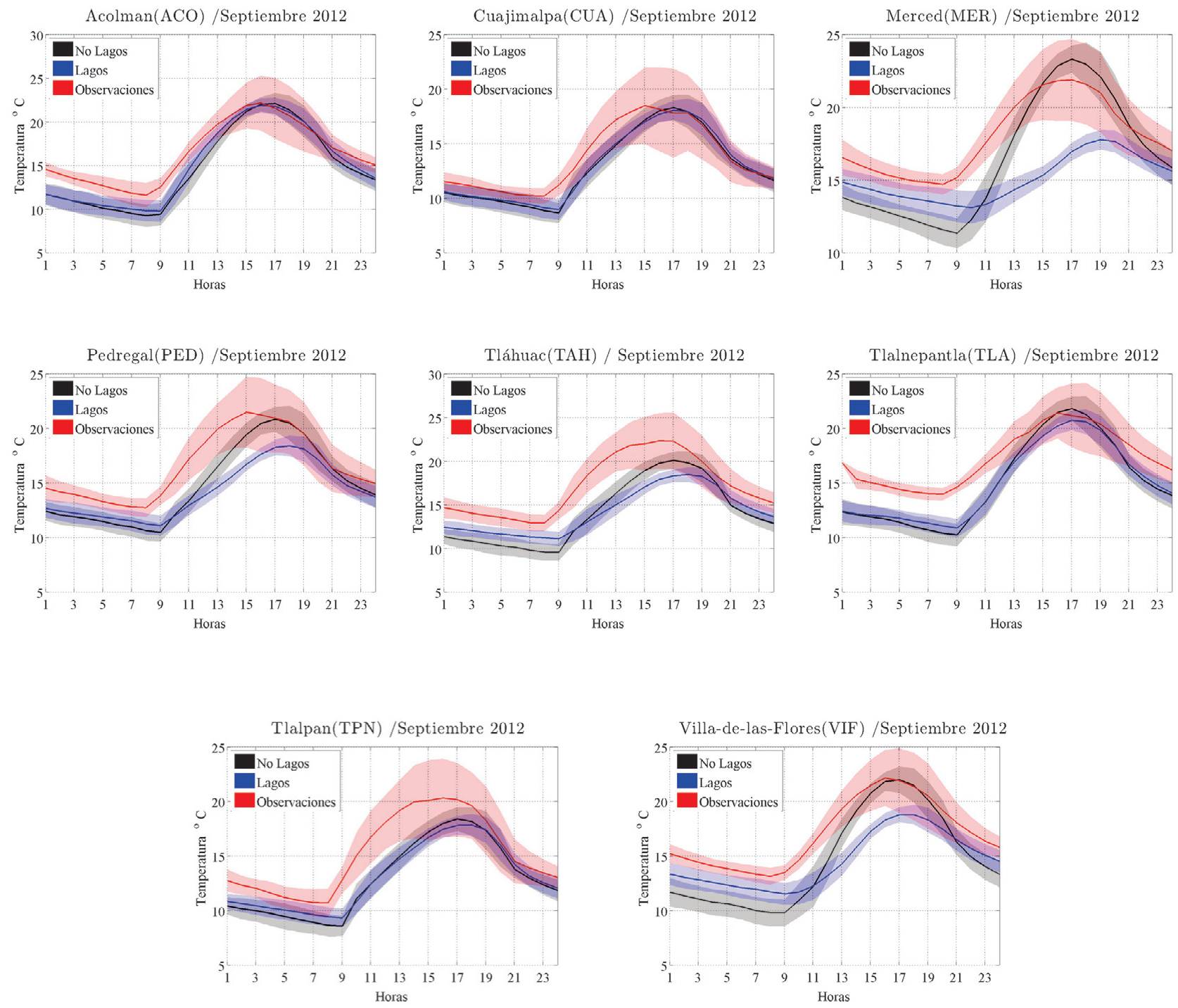

Figura 7. Comparación de las series de tiempo del promedio mensual de temperatura correspondiente al mes de septiembre de 2012. En color negro se muestra la simulación de control (No Lagos), en azul la simulación con lagos y en rojo las observaciones. El promedio mensual se representa con las líneas sólidas y sus dispersiones correspondientes con las bandas sombreadas.

Tabla 2. Relación del rango promedio y las diferencias absolutas en temperatura máxima y mínima, en grados Celsius, para las simulaciones sin lagos y con lagos. Los cálculos se muestran considerando los dos meses secos (enero-febrero) y los dos meses de lluvias (agosto-septiembre).

\begin{tabular}{|c|c|c|c|c|c|c|c|c|}
\hline & \multicolumn{4}{|c|}{$\begin{array}{c}\text { Secas } \\
\text { (enero-febrero) }\end{array}$} & \multicolumn{4}{|c|}{$\begin{array}{c}\text { Lluvias } \\
\text { (agosto-septiembre) }\end{array}$} \\
\hline & $\operatorname{Tmáx}^{0} \mathrm{C}$ & Tmín ${ }^{\circ} \mathrm{C}$ & $\begin{array}{l}\text { Rango con } \\
\text { lagos }\end{array}$ & $\begin{array}{c}\text { Rango } \\
\text { sin } \\
\text { lagos }\end{array}$ & $\operatorname{Tmáx}^{\circ} \mathrm{C}$ & Tmín ${ }^{0} \mathrm{C}$ & $\begin{array}{c}\text { Rango } \\
\text { con } \\
\text { lagos }\end{array}$ & $\begin{array}{c}\text { Rango } \\
\text { sin } \\
\text { lagos }\end{array}$ \\
\hline $\mathrm{ACO}$ & 1.41 & 0.99 & 9.32 & 11.72 & 1.1 & 0.7 & 10.74 & 12.55 \\
\hline CUA & 1.62 & 0.78 & 6.49 & 8.9 & 1.05 & 0.34 & 8.18 & 9.58 \\
\hline MER & 5.24 & 2.9 & 2.85 & 10.99 & 5.78 & 1.82 & 4.15 & 11.75 \\
\hline PED & 3.17 & 1.46 & 4.95 & 9.58 & 3.01 & 0.72 & 6.52 & 10.25 \\
\hline TAH & 2.72 & 2.18 & 5.45 & 10.35 & 2.21 & 1.65 & 6.46 & 10.31 \\
\hline TPN & 2.63 & 1.12 & 7.12 & 10.87 & 1.78 & 0.62 & 9 & 11.39 \\
\hline TLA & 1.8 & 1.05 & 6.35 & 9.2 & 1.21 & 0.7 & 7.66 & 9.58 \\
\hline VIF & 3.7 & 3.1 & 5.07 & 11.87 & 3.65 & 1.7 & 6.59 & 11.94 \\
\hline Promedio & 2.78 & 1.7 & 5.95 & 10.43 & 2.47 & 1.03 & 7.41 & 10.92 \\
\hline
\end{tabular}



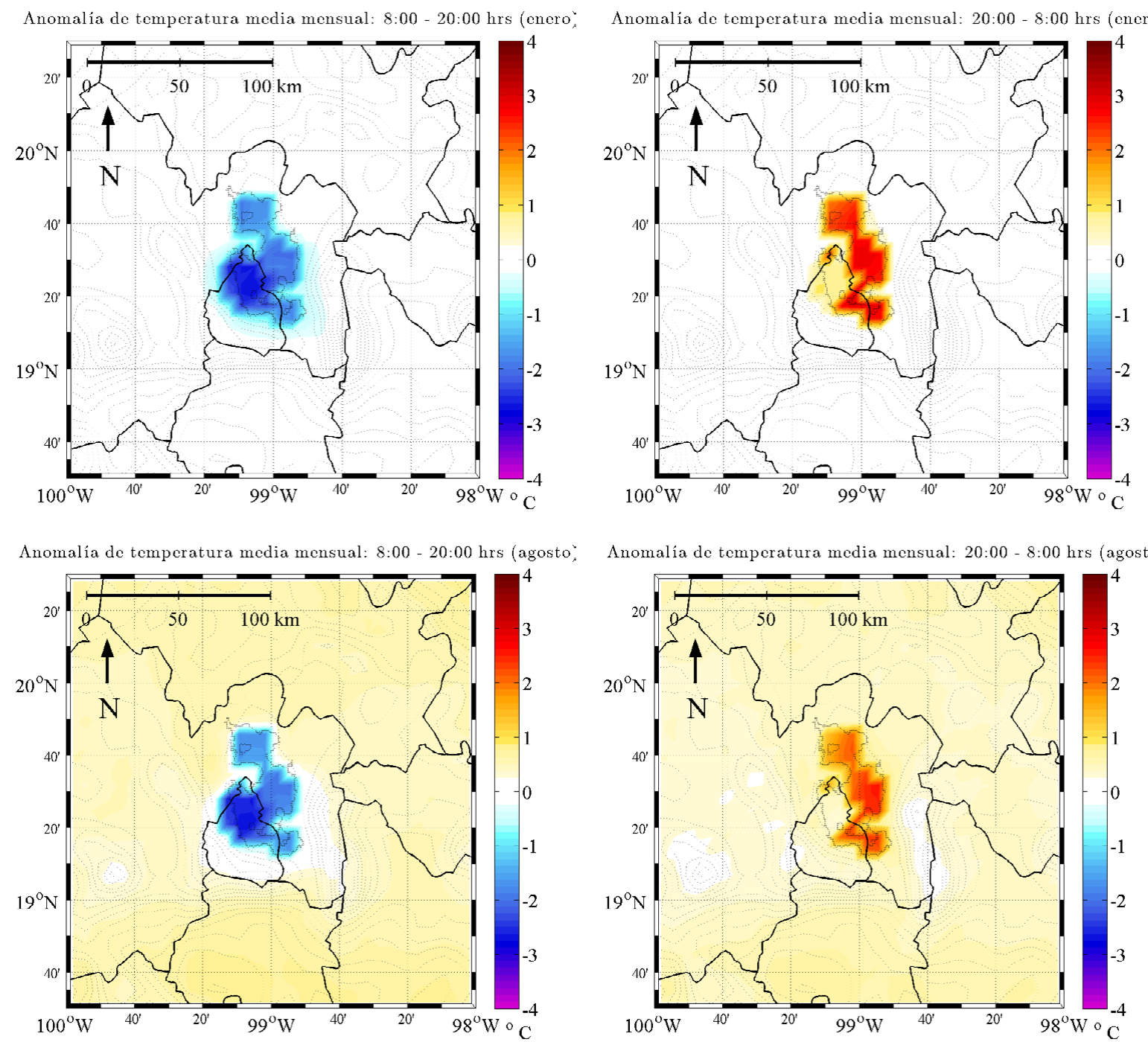

Figura 8. Mapas de las anomalías de temperatura en superficie media mensual para el día (8:00 - 20:00 horas) y para la noche (20:00 - 8:00 horas) correspondientes a los meses de enero y agosto de 2012. Las anomalías son calculadas a partir de la simulación con lagos menos la simulación de control sin lagos.

numérico ratifica que este impacto podría ser menor en un escenario con la presencia de los cuerpos de agua. Globalmente, existen diversas cuencas lacustres que enfrentan riesgos de desecación y los impactos regionales de estos cambios de uso de suelo, como se observa en este estudio, son mucho mayores que el impacto debido al cambio climático. En los modelos climáticos, el impacto, debido a la extinción de los cuerpos de agua, podría ser subestimado a causa de la baja resolución espacial. Por lo tanto, los estudios sobre los cambios de uso de suelo y su impacto regional son representados adecuadamente con modelos de predicción del tiempo de mesoescala.

\section{Agradecimientos}

Los autores agradecen el interés y el apoyo para impulsar el desarrollo de este proyecto a la Dra. Christina Siebe, al Dr. Aron Jazcilevich y al Dr. Richard Seager. Así mismo, agradecimientos al Centro de Ciencias de la Atmósfera, en especial al grupo Interacción Océano-Atmósfera por facilitar el tiempo de cómputo en el clúster. Además, agradecemos el invaluable apoyo del M.C. Octavio Gómez Ramos en la asistencia técnica para la preparación del experimento numérico. Finalmente, agradecemos las críticas constructivas de los revisores que han contribuido a enriquecer este trabajo.

\section{Referencias}

Armillas, P., 1971, Gardens on Swamps: Science, 174(4010), 653-661. Barragán, M.M., 1998, El crecimiento urbano de la Ciudad de México y la desecación del Lago de Texcoco: Relaciones, 76(XIX), 132-153. 
Dutra, E., Stepanenko, V.M., Balsamo, G., Viterbo, P., Miranda, P.M.A., Mironov, D., Schar, C., 2010, An offline study of the impact of lakes on the performance of the ECMWF surface scheme: Boreal Environment Research, 15(2), 100-112.

Endfield, H.G., 2008, Climate and Society in Colonial Mexico: A Study in Vulernability, Blackwell Publishing, $235 \mathrm{p}$.

Englehart, P.J., Douglas, A.V., 2005, Changing behavior in the diurnal range of surface air temperatures over Mexico: Geophysical Research Letters, 32, L01701.

Ezcurra, E., 1990a, De las chinampas a la megalópolis. El medio ambiente en la cuenca de México: México, Fondo de cultura económica, 85 p.

Ezcurra E., 1990b, The Basin of Mexico, en Turner, B.L. (ed.), The Earth as Transformed by Human Action, Global and Regional Changes in the Biosphere over the Past 300 Years: New York, Cambridge University Press, 577-588.

Farr, T.G., Rosen, P.A., Caro, E., Crippen, R., Duren, R., Hensley, S., Kobrick, M., Paller, M., Rodriguez, E., Roth, L., Seal, D., Shaffer, S., Shimada, J., Umland, J., Werner, M., Oskin, M., Burbank, D., Alsdorf, D., 2007, The Shuttle Radar Topography Mission, Reviews of Geophysics, 45, RG2004.

García, E. 1974, Situaciones climáticas durante la caída de la cultura Teotihuacana: Boletín Instituto de Geografía, 5, 35-70.

García, E., 1998, Climas, escala 1000000: México, Comisión Nacional para el Conocimiento y Uso de la Biodiversidad CONABIO, 1 mapa.

Global Data Assimilation System (GDAS), 2014, NCEP FNL (Final) Operational Global Analysis, 2014, disponible en $<\mathrm{http}$ ://rda.ucar. edu/datasets/ds083.2/>, accedido 10 de septiembre de 2014.

Heine, K., 1973, Variaciones más importantes del clima durante los últimos 40000 años en México, Proyecto Puebla-Tlaxcala: Comunicaciones, 7, 51-58.

Instituto Nacional de Ecología (INE), 2014, Estudio para la restauración del hábitat de las aves acuáticas migratorias, Dirección General de Protección y Restauración Ecológica: Aves Acuáticas Migratorias. AE 333 .71530 L533, $142 \mathrm{p}$, disponible en $<\mathrm{http}: / /$ repositorio.inecc. gob.mx/ae3/ae_333.71530_1533.pdf>, accedido 12 de septiembre de 2014.

Instituto Nacional de Estadística Geografía e Informática (INEGI), 2000, Estadísticas Históricas de México - Tomo 1: México, 119 p.

Intergovernmental Panel on Climate Change (IPCC), 2013, Climate Change 2013: The Physical Science Basis. Contribution of Working Group I to the Fifth Assessment Report of the Intergovernmental Panel on Climate Change, en Stocker, T.F., Qin, D., Plattner, G.K., Tignor, M., Allen, S.K., Boschung, J., Nauels, A., Xia, Y., Bex, V., Midgley, P.M. (eds.), Cambridge University Press: Cambridge, United Kingdom and New York, NY, USA, 1535 p.

Jáuregui, E., 2004, Impact of land-use changes on the climate of the Mexico City Region: Investigaciones Geográficas, Boletín del Instituto de Geografía, UNAM, 55, 46-60.

Jazcilevich, A., Fuentes, V., Jauregui, E., Luna, E., 2000, Simulated urban climate response to historical land use modification in the Basin of Mexico: Climatic Change, 44, 515-536.

Jensen, R.J., 2007, Remote Sensing of the Environment: An Earth Resource Perspective: New Jersey, Prentice Hall, 608 p.

Kalnay, E., 2003, Atmospheric Modeling, Data Assimilation and Predictability: United States of America, Cambridge University Press, $341 \mathrm{p}$.

Klaus, D., 1973, Las fluctuaciones del clima en el valle de Puebla/Tlaxcala: Comunicaciones, Proyecto Puebla-Tlaxcala, 7, 59-62.

Lankao, P.R., 2010, Water in Mexico City: what will climate change bring to its history of water-related hazards and vulnerabilities?: Environment and Urbanization, 22, 157-178.

López-Espinoza, E.D., Zavala-Hidalgo, J., Gómez-Ramos, O., 2012, Weather forecast sensitivity to changes in urban land covers using the WRF model for central Mexico: Atmósfera, 25 (2), 127-154.
Lozano-García, M.S., Ortega-Guerrero, B., Caballero-Miranda, M., Urrutia-Fucugauchi, J., 1993, Late Pleistocene and Holocene Paleoenvironments of Chalco Lake, Central Mexico: Quaternary Research, 40, 332-342.

Mancebo, F., 2007, Natural hazards and urban policies in Mexico City: Journal of alpine research, 95 (2), 108-118.

Musset, A., 1991, De l'eau vive à l'eau morte. Enjeux techniques et culturels dans la Vallée de Mexico (XVIe-XIXe s.): Paris, Éditions Recherche sur les Civilisations, $415 \mathrm{p}$.

Niederberger, B.C., 1987. Paléopaysages et archéologie pré-urbaine du bassin de Mexico, Tome 1, Collection Études mésoaméricaines 1 \& 11: Centro Francés de Estudios Mexicanos y Centroamericanos, $1-2,358,500$

Olsen, K.W., Lawrence, D.M., Bonan, G.B., Drewniak, B., Huang, M., Koven, C.D., Levis, S., Li, F., Riley, W.J., Subin, Z.M., Swenson, S., Thornton, P.E., Bozbiyik, A., Fisher, R., Heald, C.L., Kluzek, E., Lamarque, J.-F., Lawrence, P.J., Leung, L.R., Lipscomb, W., Muszala, S.P., Ricciuto, D.M., Sacks, W.J., Sun, Y., Tang, J., Yang, Z.-L., 2013, Technical Description of version 4.5 of the Community Land Model (CLM): National Center for Atmospheric Research, NCAR Technical Note, 434.

Raynal-Villasenor, J.A., 1987, The remarkable hydrological works of the Aztec civilization: Water for the Future Hydrology in Perspective: Proceeding of the Rome Symposium, 164, 3-9.

Sanders, W.T., Parsons, J.R., Stanley, R.S., 1979, The Basin of Mexico: Ecological processes in the Evolution of a Civilization: New York, Academic Press, $418 \mathrm{p}$.

Servicio Meteorológico Nacional (SMN), 2014, Temperatura máxima promedio a nivel nacional y por entidad federal, disponible en $<\mathrm{http}: / /$ smn.cna.gob.mx/climatologia/TempsyPrecip/Mensuales/2012Tmax. pdf $>$, accedido 10 de septiembre de 2014.

Sistema de Monitoreo Atmosférico (SIMAT), 2014, Bases de datos - Red de Meteorología y Radiación Solar (REDMET), disponible en $<$ http://www.aire.df.gob.mx/default.php?opc='aKBi'>, accedido 10 de septiembre de 2014.

Skamarock, W., Klemp, J.B., Dudhia, J., Gill, D.O., Barker D.M., Duda, G.M., Huang X.Y., Wang, W., Powers, G.J., 2008, A Description of the Advanced Research WRF Version 3: USA, National Center for Atmospheric Research, $113 \mathrm{p}$.

Subin, Z.M., Riley, W.J., Mironov, D., 2012, An Improved Lake Model for Climate Simulations: Model Structure, Evaluation, and Sensitivity Analyses in CESM1: Journal of Advances in Modelling Earth System 4, M02001.

Thiery, W., Stepanenko, V.M., Xing F, Klaus, D.J., Li, Z., Martynov, A., Perroud, M., Subin, Z.M., Darchambeau, F., Mironov, D., Van Lipzig, N.P.M., 2013, LakeMIP Kivu: Evaluating the representation of a large, deep tropical lake by a set of one-dimensional lake models: Tellus Series a Dynamic Meteorology and Oceanography, 66, 1-18.

Tolstoy, P., Suzanne K.F., Boksenbaum M.W., Vaughn K.B., Smith C.E., 1997, Early Sedentary Communities of the Basin of Mexico: Journal of Field Archaeology, 4, 91-106.

United States Geological Survey (USGS), 1993, Global Land Cover Characteristics Data Base Version 2.0, disponible en $<\mathrm{http}: / /$ edc2.usgs.gov/glcc/globdoc2_0.php\#dataform>, accedido $10 \mathrm{de}$ septiembre de 2014 .

Manuscrito recibido: Septiembre 12, 2014.

Manuscrito corregido recibido: Abril 14, 2015.

Manuscrito aceptado: Abril 15, 2015. 